\title{
HAMILTONIAN MECHANICS ON LIE GROUPS AND HYDRODYNAMICS
}

\author{
J. MARSDEN AND R. ABRAHAM
}

Introduction. Some of the most classical and important examples in mechanics are systems whose configuration space is a Lie group. The particular examples we have in mind are the rigid body (on the Lie group $S O(3)$ ) and the perfect fluid (on the Lie group of volume preserving diffeomorphisms).

Most of what we have to say is classical and well known. What we do is to put it in the language of global analysis with perhaps some simplification. Our sources are mainly the papers of Arnold and Blancheton [3], [4].

The paper is divided into two parts. In the first we present the general theory. In the second we describe the case of hydrodynamics. Some connections will be made with the calculus of variations in the future. In addition, a more complete exposition of the present work will appear in lecture note forms shortly [11]. ${ }^{1}$

1. Abstract theory. Let $G$ be a Lie group. ${ }^{2}$ By this we mean that $G$ is a smooth manifold modelled on a locally convex topological vector space (locally convex since, amongst other reasons, the Hahn-Banach theorem is needed) and is also a group such that group multiplication and inversion are $C^{\infty}$ mappings. The tangent bundle of $G$ is denoted $T G$ and the fiber over $x \in G$ is written $T_{x} G$.

Let $g$ be a (weak) Riemannian metric on $G$. This means that the tensor $g$ is an inner product on each $T_{x} G$, but inducing a different topology in general. For each $x \in G, e_{x} \in T_{x} G$ and $f_{x} \in T_{x} G$ we write $\left\langle e_{x}, f_{x}\right\rangle=g(x) \cdot\left(e_{x}, f_{x}\right)$.

A weak symplectic form on a manifold $M$ is a closed two form $\omega$ such that the mapping $\omega_{b}: T M \rightarrow T^{*} M$ defined by $\omega_{b}\left(e_{x}\right) \cdot f_{x}=\omega(x) \cdot\left(e_{x}, f_{x}\right)$ is injective on each fiber. (If $\omega_{b}$ is an isomorphism on each fiber, we call $\omega$ a symplectic form.)

For infinite dimensional mechanics (continuum mechanics and quantum mechanics for example), if one wishes to work with a symplectic form it is necessary to use domains for vectorfields in the same sense as occurs in semigroup theory; see [8]. If, however, one wishes to exploit differentiability directly and have the vectorfield defined and smooth in the usual sense, then it is necessary to use weak symplectic forms instead. The reason will become evident shortly. We shall use weak symplectic forms here.

Except in unusual and artificial circumstances (these can be obtained for the wave equation using the spaces in [10]), the manifolds one needs to get a smooth

\footnotetext{
'See also [14].

"The term "Lie Group" may be misleading since, in the infinite dimensional case, the usual Lie theorems do not hold. The term ILH Lie Group, or Fréchet Lie Group may be better.

${ }^{3} g$ is assumed smooth.
} 
vectorfield are usually Fréchet and not Banach, the $C^{\infty}$ functions for example. In that case the standard flow theorem for vectorfields is false. Instead one must use techniques of Browder, Kato and others. For the case of hydrodynamics with viscosity a good part of a classical book [6] is needed. Also, the flow is possibly only local, that is, cannot be extended for all time. For the nonviscous case we are concerned with, see Kato [5]. ${ }^{4}$

Let $M$ be a manifold and $\omega$ a weak symplectic form. A one form $\alpha$ can be lifted when there exists a vectorfield (unique) $X$ on $M$ such that $X^{b}=a$, where $X^{b}(m)=2 \omega_{b}(m) \cdot X(m), m \in M$. We write $X=\alpha^{*}$.

For a smooth function $f: M \rightarrow R$, such that $d f$ can be lifted, we write $X_{f}=(d f)^{*}$, and we call $X_{f}$ a Hamiltonian vectorfield.

It will be necessary to recall a few theorems about Hamiltonian systems.

Theorem 1. Let $(M, \omega)$ be a weak symplectic manifold and $X_{H}$ a Hamiltonian vectorfield with a local smooth flow $F_{r}$. Then

(i) $H \circ F_{t}=H$ (conservation of energy), and

(ii) $F_{\mathrm{r}}^{*} \omega=\omega$, or $F_{1}$ is symplectic (preserves the form $\omega$ ).

See [1] or [8] for the proof.

Recall that if $M$ is a manifold then $T^{*} M$ admits a natural weak symplectic structure given by (locally)

$$
\omega\left(\alpha_{x}\right) \cdot\left(\left(e_{1}, \alpha_{1}\right),\left(e_{2}, \alpha_{2}\right)\right)=\left[\alpha_{2}\left(e_{1}\right)-\alpha_{1}\left(e_{2}\right)\right] / 2
$$

for $e_{i} \in T_{x} M$ and $\alpha_{i} \in T_{x}^{*} M$. [ $\omega$ is symplectic iff $M$ is modelled on a semireflexive space.] See [8, Theorem 2.4$]$.

THEOREM 2. Let $G$ be a Lie group and $M$ a manifold with $\Phi: G \times M \rightarrow M a$ smooth action of $G$ on $M$, which extends naturally to an action $\Phi^{*}$ of $G$ on $T^{*} M$. Suppose $X_{H}$ is a Hamiltonian vectorfield on $T^{*} M$ and $H$ is invariant under the action $\Phi^{*}$. Then the following functions $P_{X}$ are invariant under the flow of $X_{H}$. Let $X$ be an infinitesimal generator of $\Phi$, so $X$ is a vectorfield on $M$ and define $P_{X}: T^{*} M \rightarrow R$ by $P_{X}\left(\alpha_{m}\right)=\alpha_{m}(X(m))$. (We call $P_{X}$ the momentum of $X$.)

For the proof see [8, Theorem 5.3]. This is the basic conservation law of mechanics.

In case $g$ is a weak Riemannian metric on $M$ inducing a map $g_{b}: T M \rightarrow T^{*} M$, then we deduce (using [8, Theorem 5.2]) that if $X_{n}$ is a Hamiltonian vectorfield on $T M$ (with respect to the form $\left.\omega_{g}\left(e_{x}\right) \cdot\left(\left(e_{1}, f_{1}\right),\left(e_{2}, f_{2}\right)\right)=\left[\left\langle f_{2}, e_{1}\right\rangle-\left\langle f_{1}, e_{2}\right\rangle\right] / 2\right)$ and $H$ is invariant under the induced (adjoint) action on $T M$ then the function $P_{X}\left(e_{m}\right)=\left\langle e_{m}, X(m)\right\rangle$ is invariant under the flow of $X_{n}$.

The latter situation is the one which arises naturally in the case of a Lagrangian system and will be of concern to us below.

Let us now return to the setting of Lie groups. We shall require our group and metric $g$ to have certain regularity properties introduced as follows.

\footnotetext{
4 The general nonviscous existence problem is settled in [14].
} 
Definition. ${ }^{5}$ Let $G$ be a Lie group and $g$ a weak Riemannian metric on $G$. We say that $g$ is compatible with $G$ iff

(i) $g$ is left invariant; that is, for each $x \in G, L_{x}^{*} g=g$ where $L_{x}$ is the diffeomorphism defined by $L_{x}(y)=x y$;

(ii) the kinetic energy function $T: T G \rightarrow R$ defined by $T\left(e_{x}\right)=\left\langle e_{x}, e_{x}\right\rangle / 2$ can be lifted to a (smooth) vectorfield $X_{T}$ using the weak symplectic form

$$
\omega_{9}\left(\left(e_{1}, f_{1}\right),\left(e_{2}, f_{2}\right)\right)=\left[\left\langle f_{2}, e_{1}\right\rangle-\left\langle f_{1}, e_{2}\right\rangle\right] / 2 ; \text { and }
$$

(iii) $X_{T}$ possesses a local smooth fiow (called the geodesic flow of $g$ ).

Also, we say that $G$ is a regular Lie group iff every (smooth) left invariant vectorfield on $G$ has a flow.

In the finite dimensional case these conditions are of course redundant. In the infinite dimensional case it is (iii) above which is difficult to verify.

The main conservation theorem is the following (the cases of hydrodynamics and a rigid body are due to Euler):

THEOREM 3. Let $G$ be a regular Lie group and $X_{H}$ a Hamiltonian vectorfield in $T G$ with $H$ invariant by left translations $\left(H \circ T L_{x}=H\right.$ for each $x \in G, T L_{x}: T G \rightarrow T G$ being the tangent map). Then for each $v \in T_{e} G(e=$ identity element of $G)$ the function $P_{v}$ is invariant under the flow of $X_{H}$, where

is defined by

$$
P_{v}: T G \rightarrow R
$$

$$
P_{v}\left(u_{x}\right)=\left\langle T_{e} R_{x} \cdot v, u_{x}\right\rangle
$$

where $u_{x} \in T_{x} G, R_{x}$ is right translation by $x$ and $T_{e} R_{x}$ is the tangent of $R_{x}$ evaluated at $e \in G$.

COROLLARY 4. Let $G$ be a regular Lie group and g a compatible weak Riemannian metric. Then the functions $P_{v}$ are invariant under the geodesic flow of $g$. Further, this flow is a symplectic (local) diffeomorphism and conserves (kinetic) energy.

Proof. Let $G$ act on itself by left translations. Each $v \in T_{e} G$ determines an exponential flow on $G$ by assumption, and its derivative is the infinitesimal generator $X$. Let $E_{t}$ be the exponential map of $v$. Then

$$
X(y)=d\left(E_{t}(y)\right) / d t=d\left(E_{t}(e) \cdot y\right) / d t \text { at } t=0 .
$$

Now $E_{l}(e) \cdot y=R_{y} \circ F_{t}(e)$ and so by the composite mapping theorem, $d\left(E_{l}(e) \cdot y\right) / d t$ $=T_{e} R_{y} \cdot d\left(E_{l}(e)\right) / d t=T_{e} R_{y} \cdot v$. The result is now an immediate consequence of the conservation theorem.

In practice it is usually most convenient to work in the Lie algebra $T_{e} G$ by pulling back the flow to $T_{e} G$ by left translation (in the so called "body coordinates"). The pull back of the vectorfield $X_{T}$ to $T_{e} G$ is the Euler equations. They are determined as follows:

\footnotetext{
s In the rest of section one, "left" and "right" can be interchanged, and this introduces a minus sign in Theorem 5 and Lemma 6.
} 
THEOREM 5. Let $G$ be a (regular) Lie group, $g$ a compatible metric and $X_{T}$ the corresponding Hamiltonian vectorfield with flow $F_{2}: T G \rightarrow T G$ (which may be just local). Define $H_{\text {, on }} T_{\mathrm{e}} G$ by

$$
H_{t}(v)=T_{x(t)} L_{x(t)-1} \cdot F_{t} v
$$

(where defined), where $F_{p} v \in T_{x(t)} G$. Then $H_{t}$ is a smooth flow on $T_{e} G$ and has vectorfield $Y$ uniquely determined by: $Y: T_{e} G \rightarrow T_{e} G,\langle Y(u), v\rangle=\langle[u, v], u\rangle$ where $[u, v]$ is the Lie bracket in $T_{c} G$.

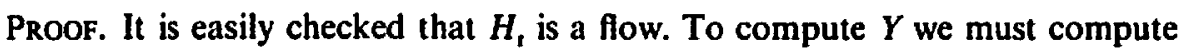
$d H_{\mathrm{t}}(v) / d t$ at $t=0$. For this we use the following:

LEMMA 6. Let $x(t)$ be a smooth curve in $G, v \in T_{e} G$ and $v(t)=\operatorname{Ad}_{x(t)-1} \cdot v$ where

$$
\operatorname{Ad}_{x}=T_{x} R_{x^{-1}} \circ T_{e} L_{x}=T_{e}\left(R_{x^{-1}} \circ L_{x}\right): T_{e} G \rightarrow T_{e} G .
$$

Then we have

$$
d v / d t=\left[v(t), T_{x(t)} L_{x(t)-1} \cdot(d x / d t)\right] .
$$

We omit the proof, as it is more or less standard; see [11] or [13].

To prove the theorem, we start with

$$
d\left\langle F_{t} u, T_{e} R_{x(t)} v\right\rangle / d t=0
$$

by the conservation law, for each $u, v \in T_{e} G$. By left invariance of $g$,

$$
\begin{aligned}
\left\langle F_{1} u, T_{e} R_{x(t)} \cdot v\right\rangle & =\left\langle T_{x(t)} L_{x(t)^{-1}} \cdot F_{t} u, \operatorname{Ad}_{x(t)-1} v\right\rangle \\
& =\left\langle H_{t} u, \mathrm{Ad}_{x(t)^{-}}, v\right\rangle .
\end{aligned}
$$

Differentiating, using Leibnitz's rule and the lemma, gives at $t=0$,

$$
\langle Y(u), v\rangle+\langle u,[v, u]\rangle=0
$$

where we use the fact that $d x / d t=F_{t} u$ (which is because we have a second order equation).

If one finds the flow of $Y$ on $T_{e} G$, the problem of finding $F_{t}$ is solved using the equation

$$
d x / d t=T_{e} L_{x(t)} \cdot H_{t} u=F_{t} u
$$

and the fact that $F_{z}$ is left invariant. For groups of diffeomorphisms and in particular for hydrodynamics, this is just a problem in ordinary differential equations.

CoRollary 7. If $v_{0}$ is a critical point of $Y$ on $T_{e} G$ then $x(t)=\exp \left(t v_{0}\right) \in G$ is the geodesic with initial value $v_{0}$.

Proof. We have $H_{1} v_{0}=v_{0}$ and so

$$
F_{1} v_{0}=T_{e} L_{x(t)} \cdot v_{0}=d x / d t .
$$

This is the equation for $x(t)=\exp \left(t v_{0}\right)$. 
By left invariance, the conservation law and conservation of energy pull back to the Lie algebra $T_{\mathrm{e}} G$.

For many circumstances it is important to work in a Hilbert space. Therefore we could complete $T_{e} G$ with respect to $\langle$,$\rangle , to obtain a Hilbert space H$. It is absolutely crucial to recognize that on $H$, the vectorfield $Y$ is not smooth and is not everywhere defined. Also, the flow $H_{t}$ is not defined on all of $H$. (This can be seen for the diffeomorphism group where $T_{\mathrm{e}} G$ corresponds to $C^{\infty}$ vectorfields, so $H$ would be $L_{2}$ vectorfields. For flows of nonsmooth vectorfields see [9].)

In the finite dimensional case, or if the energy function $T$ had $D^{2} T$ continuous on $H$ (so $Y$ would be smooth on $H$ say), then definiteness of the quadratic form $D^{2} T^{6}$ at a stationary point $v_{0}$ would imply its stability (the quadratic form is computed in Arnold [3]). Unfortunately for hydrodynamics this is not the case, so it is unknown whether or not this criterion for stability is valid, as far as we know.

2. Hydrudynamics of perfect fluids. Let $D$ be a compact orientable $n$-manifold with boundary (or without boundary). Let $g_{0}$ be a Riemannian metric on $D$ and $\Omega$ a volume (orientation) on $D$ (that is, a nonvanishing $n$-form) which is generally one derived from $g_{0}$. For tangent vectors on $D$ we write $v \cdot u$ or $\langle v, u\rangle$ for the inner product with respect to $g_{0}$.

Let $G$ be the group of volume preserving diffeomorphisms on $D$. Leslie [6], and Omori [11] show that the group of all diffeomorphisms has a structure modelled on a Fréchet space for which the group is a Lie group. The procedure has become a more or less standard one in manifolds of maps. See also [2]. A recent theorem of $D$. Ebin ${ }^{7}$ tells us that $G$ is also a Lie group (in fact a Lie subgroup). Then $T_{e} G$ may be identified with $C^{\infty}$ vectorfields $X$ on $D$ such that

(i) $\operatorname{div}_{\mathbf{\Omega}} X=0$, and

(ii) $i_{*} i_{x} \Omega=0$ where $i: \operatorname{bd}(D) \rightarrow D$ is the inclusion map.

For the full diffeomorphism group, (i) is omitted.

Condition (ii) means that $X$ is parallel to the boundary. Also, the Lie bracket in $T_{e} G$ is the negative of the usual Lie bracket of vectorfields. This requires the observation that, under an action, the map taking the Lie algebra to the infinitesimal generator is an antihomomorphism, a standard result [13]. Letting $G$ act naturally on $D$ gives the stated result.

Define a weak Riemannian structure for $G$ or all diffeomorphisms by setting

$$
\langle X, Y\rangle=\int_{D} X \cdot Y \Omega
$$

for $X, Y$ vectorfields. Extend by right translation to all of $G{ }^{8}$

CONJECTURE. ${ }^{9}$ On the regular Lie group $G,\langle$,$\rangle is compatible with G$.

${ }^{6}$ On the foliation described in [3].

' See [14] for the proof.

${ }^{8}$ For hydrodynamics one must use right invariant metrics, while it is customary to use left invariant ones for the rigid body.

${ }^{9}$ This conjecture is proven in [14]. 
That $G$ is regular is simple. Namely if $X \in T_{e} G$, and $X$ has flow $F_{v}$, then the map $f \mapsto f \circ F_{i}$ is the exponential map of $X$. This is easily seen. One cannot do this for general $X$ which are just in $L^{2}$.

For compatibility of $g=\langle$,$\rangle one must show that we get a vectorfield X$ and that it has a local flow. From $\$ 1$, it is enough to work with the Euler equations. We shall just show how to get the Euler equations $Y$ (Theorem 5). Conjecture 8 is true for the full diffeomorphism group and suitable metrics. Details may be found in [11].

THEOREM 9. For $G$ described above, the vectorfield $Y$ of Theorem 5 is given by: for each vectorfield $X$, there are $C^{\infty}$ functions $f$ and $g$ (unique up to constants) such that

$$
\widetilde{Y(X)}=-i_{X} d(\tilde{X})+d f=-L_{X} \tilde{X}+d g
$$

where $\tilde{X}$ denotes the one form obtained from $X$ via $g_{0}, i_{X}$ is the inner product, and $L_{X}$ is the Lie derivative. Traditionally $p$, defined by $p=f-\langle X, X\rangle / 2=g+\langle X, X\rangle / 2$ is called the pressure. ${ }^{10}$

Proof. First, we note that for vectorfields $X, X_{0}, Y$ that

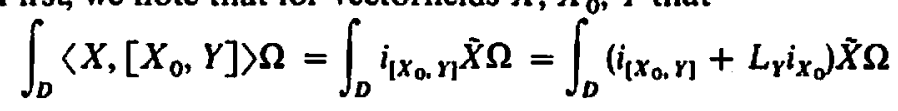

since $\int_{D} L_{Y}\left(i_{X_{0}} \tilde{X}\right) \Omega=0$ by Stoke's theorem and the boundary condition $i_{Y} \Omega=0$ on bd(D). But $i_{\left[X_{0}, Y_{1}\right.}+L_{Y} i_{X_{0}}=i_{X_{0}} L_{Y}$ (see [1]), and so we finally obtain

$$
\int_{D}\left\langle X,\left[X_{0}, X\right]\right\rangle \Omega=\int_{D} i_{X_{0}} i_{Y} d \tilde{X} \Omega=\int_{D}\left\langle Y, L_{X_{0}} \tilde{X}\right\rangle \Omega .
$$

Of course in this the fact that the vectorfields are divergence free is essential. (On the full diffeomorphism group the equations are a little different. $)^{11}$ If this condition were omitted, the pressure term would be absent.

It is a classical theorem (Hodge theory) ${ }^{22}$ that a vectorfield $Z$ can be written uniquely

$$
Z=Z_{0}+\operatorname{grad}(f)
$$

where $\operatorname{grad}(f)=d f$, and $Z_{0}$ and $\operatorname{grad}(f)$ are orthogonal and $\operatorname{div}_{\Omega} Z_{0}=0$, and $Z_{0}$ is parallel to the boundary. (The function $f$ is obtained by solving Laplace's equation $\nabla^{2} f=\operatorname{div} Z$.) One easily sees the orthogonality directly, as follows:

For any $X$ in $T_{e} G$, observe that $\langle X, \operatorname{grad}(f)\rangle=\int X \cdot \operatorname{grad}(f) \Omega=0$ since

$$
\int X \cdot \operatorname{grad}(f) \Omega=\int L_{x} f \Omega=\int L_{x}(f \Omega)=\int d i_{x}(f \Omega)=0,
$$

\footnotetext{
10 The equations for $Y$ are usually written in the equivalent form $d v / d t+\nabla_{v} v=d p$ where $\nabla$ is the covariant derivative and $v$ is an integral curve of $Y$.

"For the right invariant metric on the full diffeomorphism group, the equations are $d v / d t+v(d i v v)$ $+L_{v} v=0$. The existence problem is unknown for these equations.

$12 \mathrm{Cr}$. Morrey, Multiple integrals in the calculus of variations, Chapter 7 and also [11], [14].
} 
using Stoke's theorem and $i_{x} \Omega=0$ or bd(D). Now for the theorem we let $f$ be such that $i_{X} d(\tilde{X})-d f$ is divergence free and then observe, by our remarks, that

$$
\begin{aligned}
-\left\langle Y(X), X_{0}\right\rangle=\left\langle\left[X, X_{0}\right], X\right\rangle & =\int_{D} i_{X_{0}} i_{X} d(\tilde{X}) \Omega \\
& =\left\langle i_{X} d \tilde{X}, X_{0}\right\rangle \\
& =\left\langle i_{X} d \tilde{X}-\operatorname{grad}(f), X_{0}\right\rangle .
\end{aligned}
$$

Note that $i_{x} d(\tilde{X})$ might not be parallel to the boundary but $L_{x}(\tilde{X})$ is, and these differ by a gradient. By nondegeneracy we conclude the result.

The pressure thus constrains the motion to being divergence free.

Observe that the motion of a stationary point $X_{0}$ is just its own flow. See Corollary 7. This holds for harmonic vectorfields for example.

Finally we give a theorem classically known as Kelvin's circulation theorem. One should note that the theorem as proven in standard hydrodynamics books lacks rigor.

THEOREM 10. Let $X(t)$ be the velocity vectorfield in $T_{e} G$ above. Let I be a smooth closed loop in $D$ (a compact 1-manifold) and $l_{t}=F_{t}(l)$, where $F_{t}$ is the motion of the fluid (geodesic). Then $\mathrm{S}_{t_{c}} \tilde{X}(t)$ is independent of $t$.

PROoF. Since $F_{1}$ is a diffeomorphism,

$$
\int_{l_{t}} \tilde{X}(t)=\int_{1} F_{t}^{*} \tilde{X}(t)
$$

Now $d\left(F_{i}^{*} \tilde{X}(t)\right) / d t=F_{i}^{*} L_{X} \bar{X}+F_{i}^{*} d \bar{X} / d t$, which by Theorem 9 equals $F_{i}^{*} d g=$ $d\left(F_{t}^{*} g\right)$. Thus by Stoke's theorem,

$$
\frac{d}{d t} \int_{L_{t}} \tilde{X}(t)=\int_{1} d\left(F_{r}^{*} g\right)=0 .
$$

Curiously this is not true for the geodesic flow on the full diffeomorphism group. This theorem is quite analogous to the circulation theorem for mechanics. It also holds for $T G$, so we give it

THEOREM 11. Let $M$ be a (weak) symplectic manifold with form $\omega=d \theta$. Let $F_{8}$ be a smooth Hamiltonian flow (or local flow) on $M$. Let $A$ be a compact two manifold in $M$ with boundary $l=\operatorname{bd}(A)$, and $l_{t}=F_{l}(l)$. Then $\int_{l_{t}} \theta$ is independent of $t$.

Proof. By Stoke's theorem,

since $F_{i}^{*} \omega=\omega$.

$$
\int_{t_{t}} \theta=\int_{A_{t}} \omega=\int_{A} F_{i}^{*} \omega=\int_{A} \omega,
$$

\section{REFERENCES}

1. R. Abraham and J. Marsden, Foundarions of mechanics, Benjamin, New York, 1967.

2. R. Abraham, Lectures on global analysis, mimeographed, Princeton Univ. Press, Princeton, N.J., 1968. 
3. V. Amold, Sur la geometric differentialle des groupes de Lie de dimension infinile et ses applications à l'hydrodynamique de fluides parfaits, Ann. Inst. Fourier, (Grenoble), 16 (1966).

4. E. Blancheton, Mechanique analytique des milieus continus, Ann. Inst. H. Poincare, Sect. A, 7 (1967), 189-213.

5. T. Kato, On classical solutions of the twa dimensional non-stationary Euler equation. Arch. Rational Mech. Anal. 25 (1967), 188-200.

6. O. Ladyzhenskaya, The mathematical theory of viscous incompressible flow, Gordon and Breach, New York, 1963.

7. J. Leslie, On a differential structure for the group of diffeomorphisms, Topology, 6 (1967), 263-271.

8. J. Marsden, Hamilionion one paramerer groups, Arch. Rational Mech. Anal. 28 (1968), 362-396.

9. - Generalized Hamiltonian mechanics, Arch. Rational Mech. Anal. 28 (5), (1968), 323-361.

10. - A Banach space of analytic functions for constant coefficient equations of evolution, Canad. Math. Bull. 11 (1968).

11. _- Hamillonian mechanics, infinite dimensional Lie groups, geodesic flows and hydrodynamics, mimeographed lecture notes, University of California, Berkeley.

12. H. Omori, On the group of diffeomorphisms on a compact manifold (preprint).

13. P. Tondeur, Introduction to Lie groups and transformation groups, Springer Lecture Note Series, (1965).

14. D. Ebin and J. Marsden, Groups of diffeomorphisms and the motion of an incompressible fuid (to appear).

Universtty of California, Berkeley and Santa Cruz 